\title{
HRTEM and HRSTEM Study of Nanostructured Materials Prepared by Pulsed Laser Deposition
}

\author{
Y. T. Xing ${ }^{1}$, L.Y. Liu ${ }^{2}$, D. F. Franceschini ${ }^{1}$, W. C. Nunes ${ }^{1}$, D.J. Smith ${ }^{3}$, and I. G. Solórzano ${ }^{2}$ \\ 1. Instituto de Física, Universidade Federal Fluminense, Niterói, Brasil \\ 2. DEMa, Pontifícia Universidade Católica do Rio de Janeiro, Rio de Janeiro, Brasil \\ 3. Department of Physics, Arizona State University, Tempe, USA
}

Nanostructured materials, such as nanoparticles, thin films etc., have attracted increased attention of the scientists due to their unique properties for applications [1]. Among them, the magnetic nanoparticles are particularly interesting because of their potential wide range of possibilities for nano engineering and devices [2]. Moreover, superconducting material is very important for fundamental and theoretical studies in solid state physics [3]. In this work we studied the microstructures of Ni nanoparticles and $\mathrm{Bi} / \mathrm{Ni}$ thin films prepared by pulsed laser deposition (PLD). A JEOL JEM-ARM200CF aberrationcorrected scanning transmission electron microscope (STEM), at Lehigh, and a FEI Titan aberrationcorrected high resolution transmission electron microscope (HRTEM), at ASU, have been used to investigate the prepared nanomaterials.

Magnetic Ni nanoparticles have been prepared in $\mathrm{Ar}$ and $\mathrm{O}_{2}$ atmosphere and they show very different shape and structure as shown in Fig. 1 and 2. The cubic nanoparticle clearly shows a faceted single crystal structure with a size of $\sim 6 \mathrm{~nm}$ in which the (200) planes runs entirely across it. In contrast, the $\mathrm{Ni}$ nanoparticle prepared under $\mathrm{O}_{2}$ exhibits a spheroidal shape and a diameter of $\sim 10 \mathrm{~nm}$. The spheroidal nanoparticle contains, in fact, several crystalline clusters as lattice planes with different orientation are observed, which is very different from the single crystal cubic nanoparticle. The formation of the cubic and spherical nanoparticle is mainly due to different optimization process of nucleation rate and surface energy minimization by faceting taking place under different atmospheres.

Another sample studied in this work is a $\mathrm{Bi} / \mathrm{Ni}$ bi-layer. The thin film has been prepared at room temperature by PLD and the TEM sample has been prepared by FEI FIB system. From fig. 3 one can see clearly the deposited layers (the dark layer is the $40 \mathrm{~nm}$ Bi layer with $2 \mathrm{~nm} \mathrm{Ni}$ above) and the protecting Pt layer deposited during the sample preparation with FIB. The line scan of the Bi-Ni cross-section shows that the $\mathrm{Ni}$ and $\mathrm{Bi}$ layers are mixed already. From the high resolution image (fig. 4) one can identify that the two layers have grown epitaxial but there is not a clear defined interface between $\mathrm{Bi}$ and Ni layers, which confirms the result of fig. 3. The absence of a clear interface could be due to the interdiffusion during the deposition of $\mathrm{Ni}$ layer on top of $\mathrm{Bi}$. The superconducting properties of $\mathrm{Bi} / \mathrm{Ni}$ bi-layers, currently under investigation, is the subject of a separate report [4].

\section{References:}

[1] Y Xia, et al, Adv. Mater. 15, (2003) 353-389

[2] Q. A. Pankhurst, et al, Journal of Physics D: Applied Physics 36, (2003) R167

[3] C. P. Herzog, Journal of Physics A: Mathematical and Theoretical, 42, No 34 (2009) 34300.

[4] This research has been sponsored by CNPq (Brazil) and NSF-MWN (US: DMR-0303429) under the joint CIAM Program. The authors are grateful to PETROBRAS/ANP allowing the acquisition of the electron microscopes. 


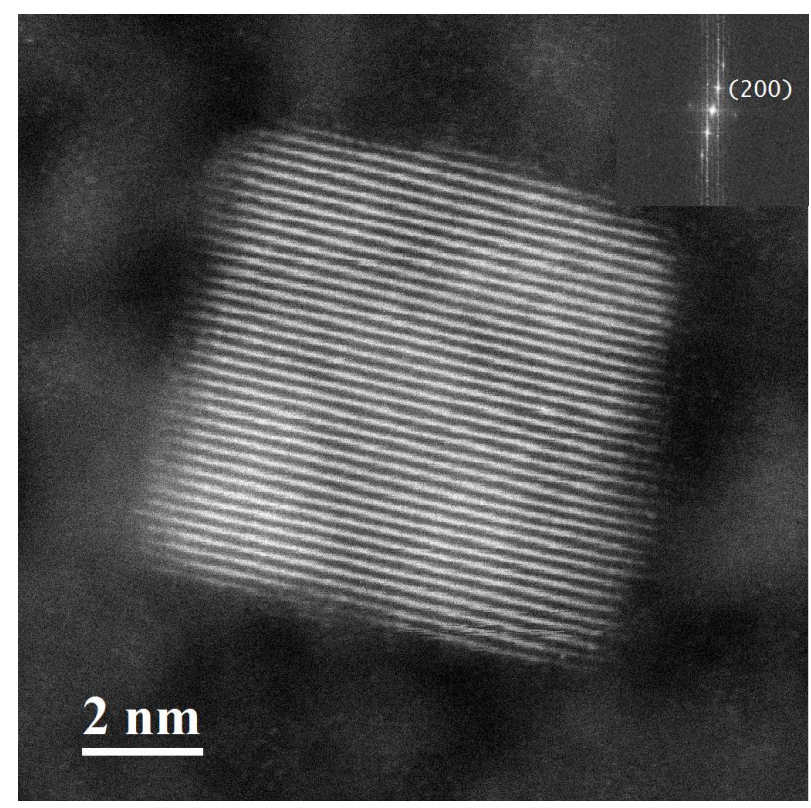

Figure 1. HRSTEM annular dark field (ADF) image of Ni nanoparticle prepared in Ar atmosphere. The (200) planes are clearly visible. Inset: FFT of the image.

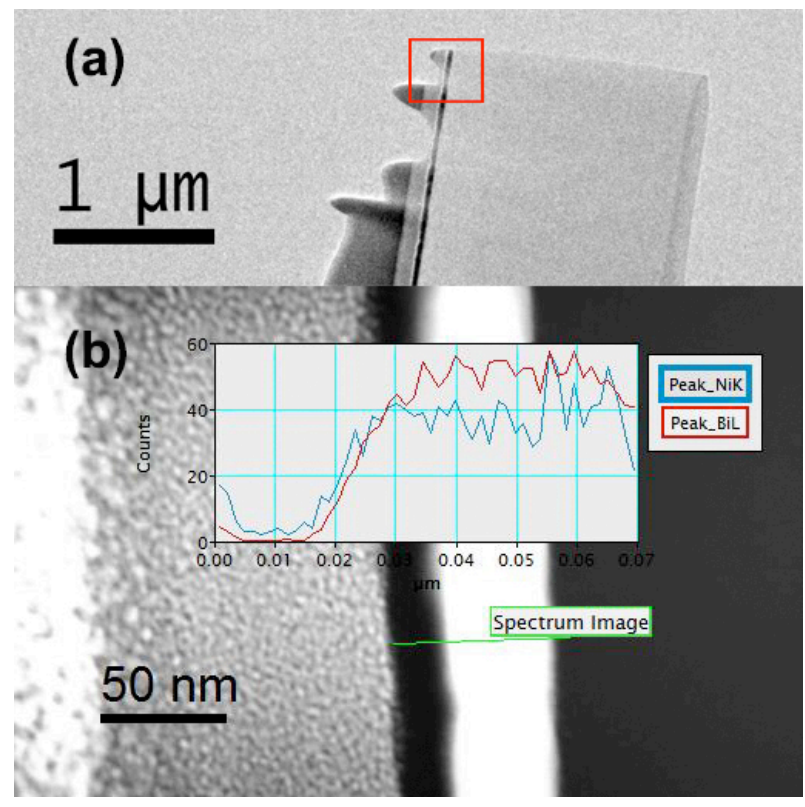

Figure 3. (a) Low magnification image of $\mathrm{Bi} / \mathrm{Ni}$ bi-layers. The sample was prepared by FIB. (b) More details of the area shown in (a) and the inset gives a line scan of the $\mathrm{Bi} / \mathrm{Ni}$ cross-section, showing that $\mathrm{Ni}$ and $\mathrm{Bi}$ are mixed.

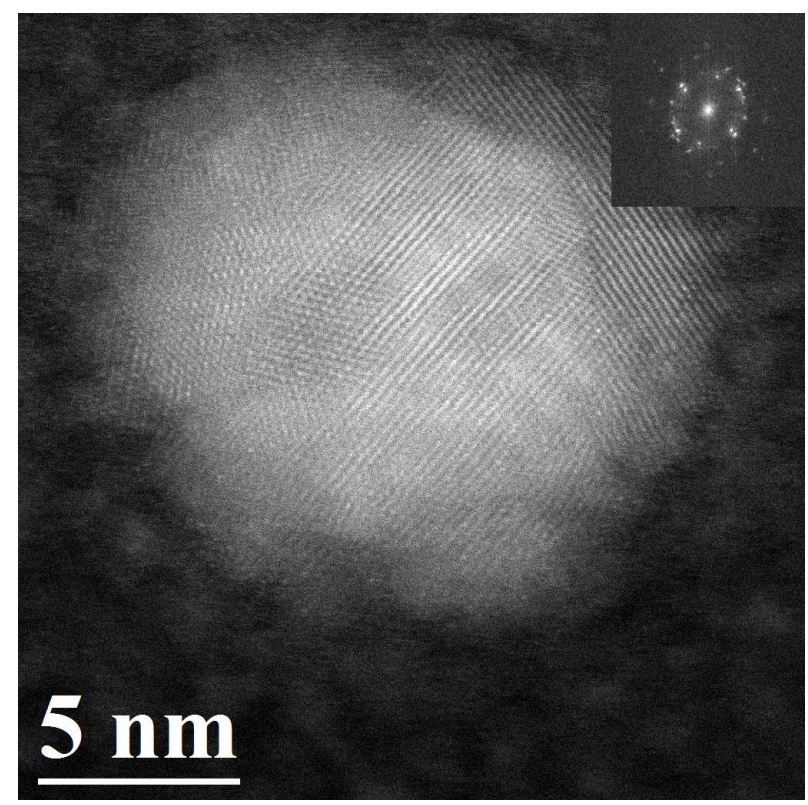

Figure 2. HRSTEM ADF image of $\mathrm{Ni}$ nanoparticle prepared in $\mathrm{O}_{2}$ atmosphere. Inset: FFT of the image.

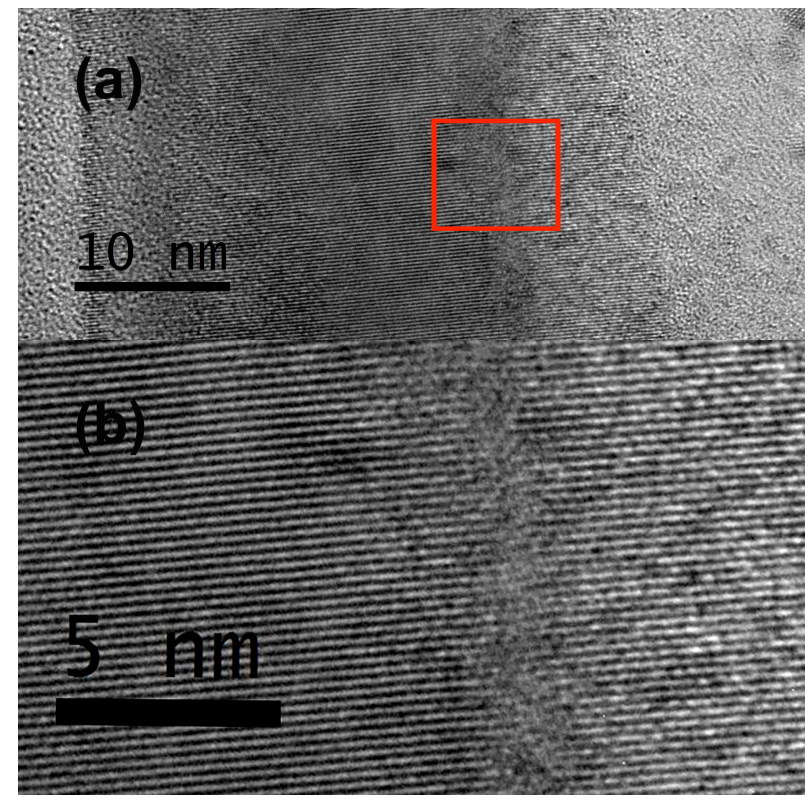

Figure 4. (a) Higher resolution image of the area showing in Fig3 (a). (b) More details of the $\mathrm{Bi} / \mathrm{Ni}$ interface. There is a very narrow disordered interface between $\mathrm{Bi}$ and $\mathrm{Ni}$. However, the $\mathrm{Bi}$ and Ni layers seem have epitaxial growth. 